\title{
Atherosclerosis and Inflammation
}

1.12 C-Reactive Protein is Associated with Aortic Stiffness Independently of Microalbuminuria in Essential Hypertension

G. Mule' (1), S. Cottone (1), P. Cusimano (1), A. Palermo (1), E. Nardi (1),

C. Geraci (1), A. Re (1), T. Bellavia (1), G. Cerasola (1)

(1)Università di Palermo, Palermo, Italy

Introduction. Previous studies documented an association between microalbuminuria (MAU) and low grade chronic inflammation, expressed chiefly by elevated plasma levels of high-sensitivity Creactive protein (HS-CRP). A link between both MAU and HS-CRP and an increased aortic stiffness has also been demonstrated. However, it has not been fully elucidated if the relationships of MAU and HS-CRP with aortic distensibility are independent from each other.

Aim. To evaluate the relationships of urinary albumin excretion rate (AER), HS-CRP and aortic stiffness in a sample of hypertensive patients without over renal insufficiency.

Methods. We enrolled 112 untreated non-diabetic essential hypertensive patients (mean age: $45 \pm 11$ years; males $68 \%$ ). In all subjects 24-hour AER and HS-CRP were determined by immunoenzymatic assay. Subjects with AER $\geq 20 \mu \mathrm{g} / \mathrm{min}$ were considered microalbuminuric. Aortic stiffness was assessed by carotid-femoral PWV (c-f PWV) measurement using the Complior, a computerized device that allows on-line pulse wave recording and automatic calculation of PWV.

Results. C-f PWV values were higher in microalbuminuric subjects $(n=46)$ than in normoalbuminuric ones $(\mathrm{n}=66)(11.8 \pm 2.2 \mathrm{vs} 10.1 \pm 1.9 \mathrm{~m} / \mathrm{sec} ; \mathrm{p}<0.001)$ and in subjects with HS-CRP above the median value $(1.8 \mathrm{mg} / \mathrm{l})$ when compared to those with lower levels $\mathrm{f}$ HS-CRP $(11.7 \pm 2.3 \mathrm{vs} 10 \pm 1.8$ $\mathrm{m} / \mathrm{sec} ; \mathrm{p}<0.001)$. These differences remained statistically significant even after adjustment for age, sex and mean blood pressure ( $<<0.001$ for MAU and $p=0.038$ for HS-CRP). In a multiple regression model in which c-f PWV was considered as dependent variable, and age, gender, MAP, waist circumference, glycaemia, triglycerides, HDL cholesterol, total cholesterol and smoking habit were included as covariates, along with AER and HS-CPR, both these latter parameters remained independent predictors of aortic stiffness $(r=0.24 ; p<0.001$ and $r=0.14 ; p=0.026$, respectively).

Conclusions. Our study seems to suggest that in patients with essential hypertension both microalbuminuria and C-reactive protein are independently associated with an increased aortic stiffness. The strength of their associations with carotid-femoral PWV is greater for albumin excretion rate than for HS-CPR. 